\title{
Minimum wave-localization length in a one-dimensional random medium
}

\author{
Ping Sheng, Benjamin White, Zhao-Qing Zhang, ${ }^{*}$ and George Papanicolaou ${ }^{\dagger}$ \\ Corporate Research Science Laboratories, Exxon Research and Engineering Company, Clinton Township, \\ Route 22 East, Annandale, New Jersey 08801
}

(Received 16 May 1986)

\begin{abstract}
The frequency dependence of the localization length $l$ for acoustic and electromagnetic waves in a one-dimensional randomly layered medium is studied both numerically and analytically. Through the consideration of different types of random-media models characterized by abrupt or continuous variation of the material parameters, it is shown that beyond the low-frequency behavior of $l \sim \omega^{-2}$, where $\omega$ denotes the angular frequency, the localization length either approaches a constant or diverges at high frequencies. In all cases, the value of $l$ for a given random medium is found to exhibit a well-defined lower bound whose value is generally several orders of magnitude times the correlation length of the inhomogeneities. The dependence of this minimum localization length on the amount of randomness, plus a comparison with the Schrödinger wave-localization length behavior, are presented and discussed.
\end{abstract}

Localization is a phenomenon generic to waves in random media. ${ }^{1-4}$ With systems of less than two spatial dimensions, for example, it is now known that all waves, Schrödinger or classical (electromagnetic and elastic), are localized with an infinitesmal amount of randomness. ${ }^{1,2}$ While the basic physics of localization is similar for Schrödinger and classical waves, the differences in the dispersion relation and the wave boundary conditions imply that with regard to specific behaviors, such as the wave-propagation characteristics and the frequency dependence of the localization length, the two cases are expected to be distinct. Recent studies ${ }^{2-4}$ of the localization length in the low-frequency regime have shown that, whereas a quantum particle generally becomes more localized in a disordered medium as its energy decreases, the opposite is the case for classical waves since a lowfrequency wave tends to perceive the disordered system as an essentially homogeneous effective medium due to its poor resolving capability. In this work we study classical wave localization in a one-dimensional continuous random medium and point out that at intermediate and high frequencies the localization length variation differs significantly from that of the low-frequency regime. In particular, we show that for a wide class of random material parameter variations the localization length of a given medium is always characterized by a well-defined minimum value, which is generally orders of magnitude larger than the correlation length of the inhomogeneities. Furthermore, depending on whether the random variations occur abruptly or continuously the minimum value either is approached asymptotically at high frequencies or occurs at some intermediate frequency. Comparison with the Schrödinger wave-localization behavior indicates that there is no comparable counterpart to the minimum localization length in the quantum particle case.

Consider a one-dimensional medium with spatially varying density $\rho(z)$, elastic modulus $K(z)$, dielectric constant $\epsilon(z)$, and magnetic permeability $\mu(z)$. Since in one dimension the elastic wave equation and the electromag- netic wave equation are related by a mapping of the variables, ${ }^{5}$ the results obtained in one case are ensured to be applicable to the other case as well. In this work we will use the notations of the elastic case. The wave equation can be written as a pair of coupled first-order differential equations:

$$
\begin{aligned}
& \rho(z) \frac{\partial W}{\partial t}=-\frac{\partial P}{\partial z}, \\
& \frac{\partial P}{\partial t}=-K(z) \frac{\partial W}{\partial z},
\end{aligned}
$$

where $W$ denotes displacement velocity and $P$ the pressure. In frequency domain, where we let $\exp (-i \omega t)$ be the time dependence of $W$ and $P$, the equation becomes

$$
\frac{d}{d z}\left(\begin{array}{l}
P \\
U
\end{array}\right)=\omega\left(\begin{array}{cc}
0 & \rho(z) \\
-1 / K(z) & 0
\end{array}\right)\left(\begin{array}{l}
P \\
U
\end{array}\right),
$$

where $U \equiv i W$. We would first like to show that for random $\rho$ and $K$, the localization length $l$ of the elastic wave is nondecreasing at high frequencies.

Localization of the wave means that the dominant behavior for the solution to Eq. (2) must decay like $\exp (-\gamma z)$ as $z \rightarrow \infty$, where $\gamma \equiv l^{-1}$. However, the fact that the matrix on the right-hand side of Eq. (2) has zero trace implies that there must also be a linearly independent solution which grows like $\exp [+\gamma z]$ with exactly the same value of $\gamma$. For arbitrary choice of the initial condition, the growing solution will be picked up with probability one. We thus take $P(0)=1$ and $U(0)=0$. By making a coordinate transformation $P=r \cos \theta, U=r \sin \theta$, Eq. (2) takes the form

$$
\begin{aligned}
& \frac{d \theta}{d z}=-\omega\left[\rho(z) \sin ^{2} \theta+K^{-1}(z) \cos ^{2} \theta\right], \\
& \frac{d r}{d z}=(r \omega / 2) \sin (2 \theta)\left[\rho(z)-K^{-1}(z)\right],
\end{aligned}
$$


with $r(0)=1$ and $\theta(0)=0$. The $\theta$ equation is now decoupled from the $r$ equation, and the value of $\gamma$ can be expressed as

$\gamma=\lim _{z \rightarrow \infty} \frac{1}{z} \ln r=\frac{\omega}{2} \lim _{z \rightarrow \infty} \frac{1}{z} \int_{0}^{z} \sin (2 \theta)\left[\rho(x)-K^{-1}(x)\right] d x$.

We recognize from Eq. (4) that $\gamma$ is essentially the average of $\sin (2 \theta)\left(\rho-K^{-1}\right)$. To carry out this averaging, we assume that $\rho=\rho[\xi(z)], K=K[\xi(z)]$, where $\xi(z)$ is a stationary, ergodic, Markov process ${ }^{6}$ with infinitesmal generator $Q$. Since we allow $\xi(z) \in \mathbf{R}^{d}$ to have a state space of arbitrarily large dimension $d$, a very large class of random models is included in this formulation. From the assumed properties it follows that $\xi(z)$ has an invariant distribution function $\bar{P}(\xi)$ which satisfies the Fokker-Planck equation $Q^{*} \bar{P}(\xi)=0$, with $Q^{*}$ denoting the adjoint of $Q$. The ergodic theorem then tells us that the averaging expressed in Eq. (4) can alternatively be written as

$\gamma=\frac{\omega}{2} \int_{0}^{2 \pi} d \theta \int_{\mathbf{R}^{d}} d \xi P(\theta, \xi) \sin (2 \theta)\left[\rho(\xi)-K^{-1}(\xi)\right]$.

Here $P(\theta, \xi)$ is the joint invariant measure for the parameter set $(\theta, \xi)$ which completely describes the Markov process for the $r$ variable. $P(\theta, \xi)$ satisfies the equation $\mathscr{L}^{*} P(\theta, \xi)=0$, where from Eq. (3a)

$$
\mathscr{L}=Q-\omega\left(\rho \sin ^{2} \theta+K^{-1} \cos ^{2} \theta\right) \frac{\partial}{\partial \theta}
$$

is the infinitesmal generator for $(\theta, \xi)$. Since the knowledge of $P(\theta, \xi)$ can directly yield $\gamma$ through Eq. (5), we now focus our effort on getting $P(\theta, \xi)$ at the highfrequency limit. Let $P$ be expanded as

$$
P=P^{(0)}+\frac{1}{\omega} P^{(1)}+\frac{1}{\omega^{2}} P^{(2)}+\cdots
$$

for $\omega \rightarrow \infty$. Substitution of Eq. (7) into Eq. (5) yields $\gamma=c_{0} \omega+c_{1}+c_{2} / \omega+\cdots$ for the asymptotic behavior of $\gamma$. The equation $\mathscr{L}^{*} P(\theta, \xi)=0$ can now be expressed as

$$
\begin{aligned}
& \frac{\partial}{\partial \theta}\left[\left(\rho \sin ^{2} \theta+K^{-1} \cos ^{2} \theta\right) P^{(0)}\right]=0, \\
& Q^{*} P^{(n)}+\frac{\partial}{\partial \theta}\left[\left(\rho \sin ^{2} \theta+K^{-1} \cos ^{2} \theta\right) P^{(n+1)}\right]=0,
\end{aligned}
$$

where $n=0,1,2, \ldots$. From Eq. (8a) and the requirement that

$$
\int_{0}^{2 \pi} d \theta P(\theta, \xi)=\bar{P}(\xi),
$$

we get

$P^{(0)}(\theta, \xi)=\left[\frac{\rho(\xi)}{K(\xi)}\right] \frac{\bar{P}(\xi)}{2 \pi\left[\rho(\xi) \sin ^{2} \theta+K^{-1}(\xi) \cos ^{2} \theta\right]}$

Substitution of Eq. (10) into Eq. (5) shows that the contribution from $P^{(0)}(\theta, \xi)$ vanishes because the $\theta$ integration gives zero. That means $\gamma \sim c_{1}+c_{2} / \omega+\cdots$ as $\omega \rightarrow \infty$, which proves our assertion that localization length is nondecreasing at high frequencies. The next term of expansion in $P(\theta, \xi), P^{(1)}$, can be obtained from Eq. (8b) by using $P^{(0)}$, Eq. (10), as the input. Insertion of the resulting $P^{(1)}$ into Eq. (5) yields a general expression for $c_{1}$ :

$$
c_{1}=-\frac{1}{4 \pi} \int_{\mathbf{R}^{d}} d \xi \bar{P}(\xi) \int_{0}^{2 \pi} d \theta \sin (2 \theta) \arctan \left\{[\rho(\xi) K(\xi)]^{1 / 2} \tan \theta\right\} Q\left(\frac{\rho(\xi)-K^{-1}(\xi)}{\rho(\xi) \sin ^{2} \theta+K^{-1}(\xi) \cos ^{2} \theta}\right)
$$

where the arctan function is understood to be the branch which is zero when $\theta=0$, and is monotonically increasing.

To obtain the localization length behavior for all frequencies, we have to consider specific random-media models. For the first model we will let $\xi(z)=\left(\xi_{1}, \xi_{2}\right)$ and

$$
\begin{aligned}
& \rho=1+2 \sigma_{\rho} \xi_{1}(z), \\
& K^{-1}=1+2 \sigma_{K} \xi_{2}(z),
\end{aligned}
$$

where $0<\sigma_{\rho(K)}<1$ gives the amplitude of randomness, and $\xi_{1}, \xi_{2}$ are random step functions which jump simultaneously and whose values are independent and uniformly distributed in $\left[-\frac{1}{2}, \frac{1}{2}\right]$. The thickness $\Delta z$ of a given layer (in which the value of $\xi_{1(2)}$ is constant) is assumed to be exponentially distributed with the probability density $\bar{a}^{-1} \exp (-\Delta z / \bar{a}), \bar{a}$ being the mean layer thickness. In what follows we will let $\bar{a}=1$. It is clear that in the present case $\bar{P}(\xi)=1$ on a square $\left[-\frac{1}{2}, \frac{1}{2}\right] \times\left[-\frac{1}{2}, \frac{1}{2}\right]$, and the operation of $Q$ represents
$Q f\left(\xi_{1}, \xi_{2}\right)=\int_{-1 / 2}^{1 / 2} d z_{1} \int_{-1 / 2}^{1 / 2} d z_{2}\left[f\left(z_{1}, z_{2}\right)-f\left(\xi_{1}, \xi_{2}\right)\right]$.

We note that the present model can easily be adapted to the special case in which the values of $\xi_{1}, \xi_{2}$ are binary in nature (two different materials). The mathematics actually simplifies in that instance. Equations (11)-(13) complete the prescription for calculating the high-frequency behavior of the model. For low frequencies, the limiting form of $\gamma$ has been determined by previous works ${ }^{2-4}$ to be $\gamma \sim \omega^{2}$. For completeness, we write down the lowfrequency asymptotic expression for $\gamma$ with all the pertinent factors:

$$
\gamma=\frac{1}{12}\left(\sigma_{\rho}^{2}+\sigma_{K}^{2}\right) \bar{a} v_{0}^{-2} \omega^{2}, \omega \rightarrow 0
$$

where $v_{0} \equiv\left(K_{0} / \rho_{0}\right)^{1 / 2}=1$ is the average velocity of the medium. To interpolate between the low- and the highfrequency limits, we use transfer matrix to obtain numerically simulated values of $\gamma$. That is, 


$$
\gamma=\left\langle\frac{1}{z} \ln T(z)\right\rangle,
$$

where \langle\rangle denotes configurational averaging, $T=\left(1-|R|^{2}\right)^{1 / 2}$, with $R=-q^{*} / p^{*}$, and $p, q$ are given by

$$
\left[\begin{array}{cc}
p & q \\
q^{*} & p^{*}
\end{array}\right)=\prod_{i=0}^{N} \mathscr{M}_{i+1, i}
$$

The notation $\mathscr{M}_{i+1, i}$ denotes a $2 \times 2$ transfer matrix with elements

$$
\begin{aligned}
m_{11}=m_{22}^{*}= & 0.5\left\{1+\left[\left(\rho_{i+1} K_{i+1}\right) /\left(\rho_{i} K_{i}\right)\right]^{1 / 2}\right\} \\
& \times \exp \left[i \omega\left(\phi_{i+1}+\phi_{i}\right)\right] \\
m_{12}=m_{21}^{*}= & 0.5\left\{1-\left[\left(\rho_{i+1} K_{i+1}\right) /\left(\rho_{i} K_{i}\right)\right]\right\}^{1 / 2} \\
& \times \exp \left[i \omega\left(\phi_{i+1}-\phi_{i}\right)\right]
\end{aligned}
$$

and $\phi_{i}=a_{i}\left(\rho_{i} / K_{i}\right)^{1 / 2} / 2$, where $\rho_{i}$ and $K_{i}$ are, respectively, the density and elastic modulus of $i$ th layer, and $a_{i}$ is its thickness. In Fig. 1 we plot the numerically calculated localization length $l\left(=\gamma^{-1}\right)$ as a function of wavelength $\lambda$ $(=2 \pi / \omega)$, both in units of $\bar{a}(=1)$, for three values of $\sigma=\sigma_{\rho}=\sigma_{K}$. Every point represents the result of averaging over 40 configurations. The low-frequency limit, evaluated from Eq. (14), and the high-frequency limit, evaluated from Eqs. (11)-(13), are also shown by dashed lines. It is seen that except for statistical fluctuations, the behavior of the localization length is essentially a smooth interpolation between the low- and the high-frequency limits. The minimum localization length for this model is therefore given by $1 / c_{1}$. On the same graph we also show the localization length for the quantum Schrödinger wave where the random parameter is the potential $V$ :

$$
\bar{a}^{2} \frac{2 \mu V}{\hbar^{2}}=\sigma_{s}[1+2 \xi(z)]
$$

where $\mu$ is the particle mass, $\hbar$ is Planck's constant, and the wavelength is defined by $\lambda=2 \pi \hbar / \sqrt{2 \mu E}$ (the zero of $E$ in this case is defined by the lower bound of the potential, which also corresponds to the lower bound for the energy eigenvalues). The localization length in this case is again calculated by the transfer matrix method with each point representing averaging over 50 configurations. Besides the trend of the high-frequency variation being dissimilar to that of the classical wave case, the localization length for a quantum particle clearly has no lower bound. One source of these differences can be traced to the dispersion relations of the waves and their effect on the reflection coefficient at an interface: whereas the classical wave reflection coefficient is independent of the frequency, the quantum particle always becomes more transmitting as its energy increases. Another source of difference is that whereas for the quantum particle with small $E$ there can be regions where $V>E$ and the Schrödinger wave becomes exponentially attenuated, the acoustic wave in a nondissipative continuous medium is always (locally) propagating in nature. However, it should be remarked that for the electromagnetic wave in a medium containing metallic elements $(\epsilon<0)$ the analogy with the acoustic

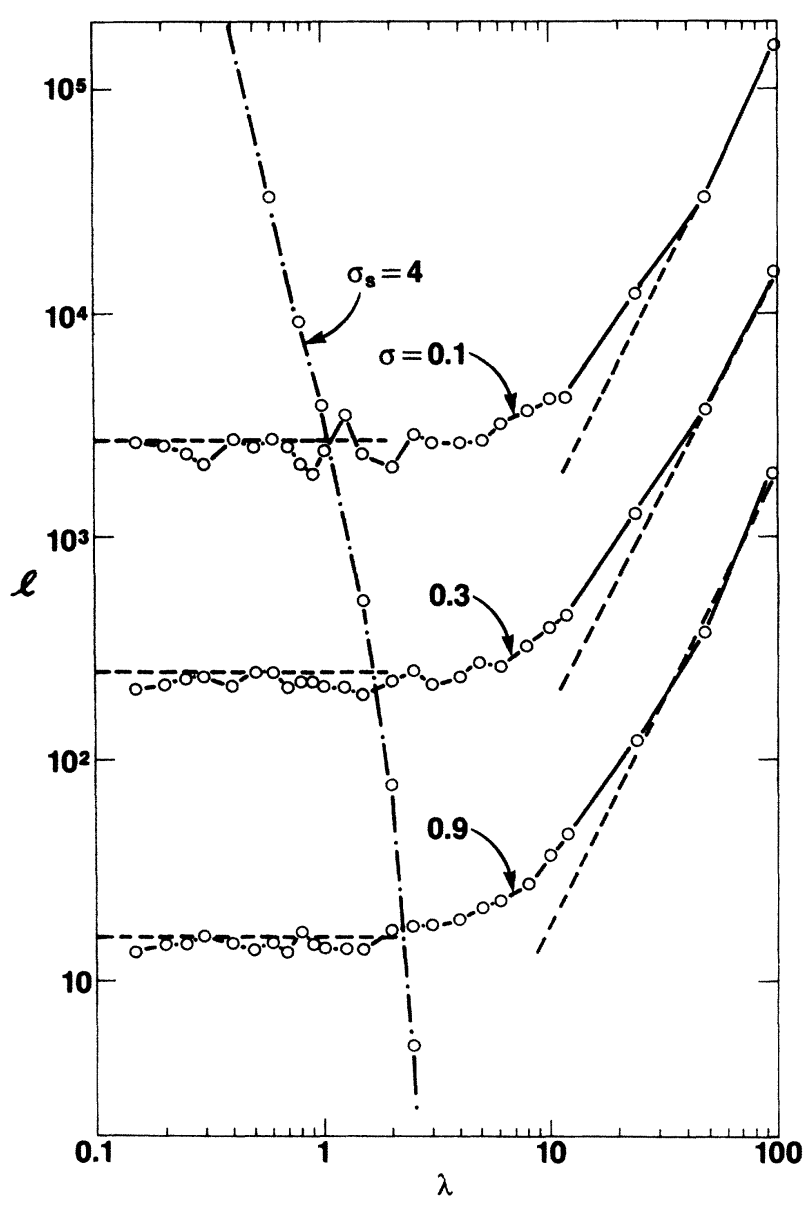

FIG. 1. Localization length for the discontinuous model plotted as a function of wavelength $\lambda=2 \pi / \omega$ (mean velocity is 1 in the present case) for three values of the randomness parameter $\sigma$. Dashed lines denote high- and low-frequency limiting behaviors evaluated from Eqs. (11)-(14). The localization length for a quantum particle is shown by the dashed-dotted line. Both the localization length and the wavelength are measured in units of average layer thickness $\bar{a}$, taken to be 1 in the present case.

wave breaks down (as pointed out in Ref. 5), since there the wave can become exponentially attenuated inside regions of negative $\epsilon$. In that particular case a different mathematical treatment is needed, and the result obtained so far in regard to the localization length lower bound are not expected to be applicable.

The second model we would like to consider is one where there is no abrupt variation of the material parameters. One way to obtain such a model is to smooth the material parameters of the first model with an exponential filter. The discontinuities in the material parameters then becomes discontinuities in the first derivative of the material parameters with respect to $z$. More precisely, if we let $\bar{m}$ denote the material parameter $\rho$ or $K^{-1}$ of the first model and $m$ denote that of the second model, then

$$
\frac{d m}{d z}=-\alpha m+\beta \bar{m}
$$

so that 


$$
m(z)=\beta \int_{-\infty}^{t} \exp [-\alpha(z-s)] \bar{m}(s) d s,
$$

where $\alpha$ and $\beta$ are constants. Since $\bar{m}$ is a piecewise constant function, Eq. (18) can be integrated within each constant region to obtain

$$
m(z)=\exp \left(z_{1}-z\right) m\left(z_{1}\right)+\left[1-\exp \left(z_{1}-z\right)\right] \bar{m},
$$

where $z_{1}<z$ denotes the coordinate for the lower end of the layer, and we have let $\alpha=\beta=1$ so that $\langle m\rangle=\langle\bar{m}\rangle$. By using this model, the calculation of the asymptotic leading term of $\gamma$ yields $c_{1}=0$. One immediate implication is that instead of approaching a constant, the localization length now increases at high frequencies. Since it is known that in the low-frequency regime the localization length increases as the frequency decreases, there must be a minimum at some intermediate frequency. By subdividing each layer into ten intervals, we have carried out numerical simulation on the continuous model by treating the material parameters within each interval as piecewise constant and using the transfer matrix to calculate $\gamma$. In Fig. 2 the calculated localization length for the three $\bar{m}$

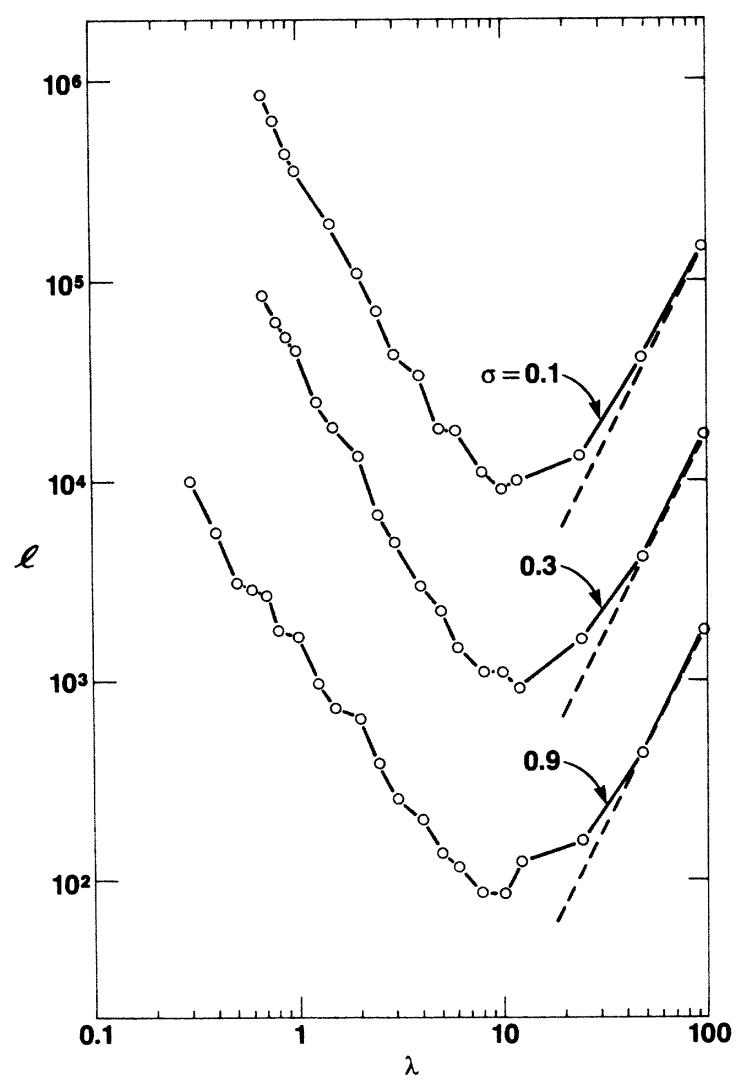

FIG. 2. Localization length for the continuous model plotted as a function of wavelength $\lambda=2 \pi / \omega$ (mean velocity is 1 in the present case). The three values of $\sigma$ denote the amount of randomness used to generate the $\bar{m}$ parameters. The material parameters for the continuous model is obtained from $\bar{m}$ by using Eq. (19). The units for localization length and wavelength are the same as those in Fig. 1. The dashed lines denote the lowfrequency limiting behavior given by Eq. (14). parameter sets shown in Fig. 1 is plotted as a function of wavelength. Each point is noted to be the average for 40 configurations. The minimum of the localization length is seen to occur at $\lambda \simeq 10 \bar{a}$, with the behavior for low frequencies, $\lambda \gg 10 \bar{a}$, approaching that given by Eq. (14) (shown by dashed lines). Physically, the divergence of localization length at high frequencies is understandable in terms of decreased reflection coefficient since, as the wavelength decreases, the wave begins to perceive the environment as slowly varying and the wave transmission is consequently increased. We expect a similar effect to occur for the Schrödinger wave if the variation of $V$ becomes smooth. However, that would not alter the qualitative behavior of the Schrödinger wave-localization length as a function of frequency. An interesting (hypothetical) problem arises if we consider the electromagnetic wave and allow the presence of metallic regions within the context of the continuous-variation model. Can the highfrequency divergence of $l$ be counteracted by the presence of negative $\epsilon$ regions? If so, for a given fraction of metallic elements how negative would $\epsilon$ have to be before the high-frequency trend of $l$ is reversed? We have no clearcut answers to these questions at present.

In Fig. 3 the values of minimum localization length $L$

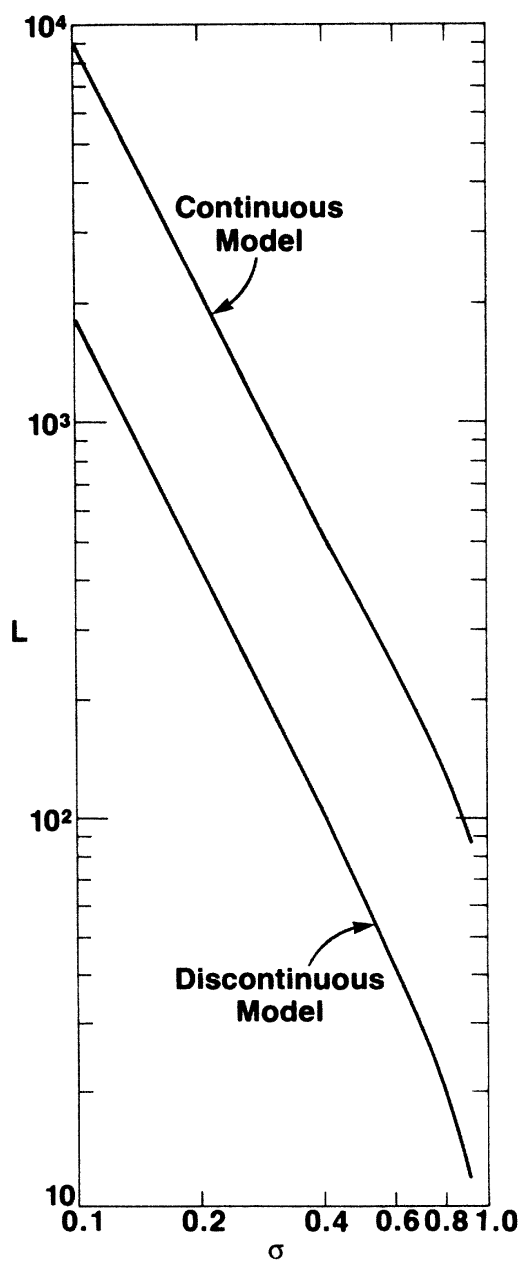

FIG. 3. Minimum localization length for the discontinuous and the continuous models plotted as a function of $\sigma$. 
for the two models are plotted as a function of randomness parameter $\sigma\left(=\sigma_{\rho}=\sigma_{K}\right)$. It is noted that the variation of $L$ can be approximately described by the relation

$$
L \propto \sigma^{-\delta},
$$

where $\delta$ varies from 2 at small $\sigma$ to $\gtrsim 3$ for $\sigma \rightarrow 1$.

Besides the two types of random-media model considered above, we have also numerically simulated a nonMarkovian model in which $\rho$ and $K^{-1}$ are given by Eq. (12) but the layer thickness is constant. While $\gamma$ still behaves as $\omega^{2}$ at low frequencies, at intermediate frequencies the localization length displays oscillations before set- tling down to a constant at high frequencies. The oscillations are interpretable as a remnant of a periodic system where there are bands of delocalized states. In this case the localization length minimum occurs at some intermediate frequency.

In summary, we have shown that under the assumption of Markov, stationary, and ergodic dependence for the random material parameters the localization length for classical waves (with special case of exception noted) in one dimension is nondecreasing at high frequencies and generally possesses a minimum value. Implications of this result, plus consideration of localization in higher dimensions, are presently being pursued.
*On leave from San Francisco State University, 1600 Halloway, San Francisco, California 94132, and Institute of Physics, Academia Sinica, People's Republic of China.

†Permanent address: Courant Institute of Mathematical Sciences, 251 Mercer Street, New York, New York 10012.

${ }^{1}$ E. Abrahams, P. W. Anderson, D. C. Licciardello, and T. V. Ramakrishnan, Phys. Rev. Lett. 42, 673 (1979).

${ }^{2}$ S. John, H. Sompolinsky, and M. J. Stephen, Phys. Rev. B 27, 5592 (1983).

${ }^{3}$ M. Y. Azbel, Phys. Rev. B 28, 4106 (1983).

${ }^{4}$ W. Kohler and G. Papanicolaou, J. Math. Phys. 14, 1733 (1973).
${ }^{5} \epsilon / c \rightarrow \rho, c / \mu \rightarrow K$, electric field $\rightarrow$ displacement velocity, magnetic field $\rightarrow$ pressure. Here $c$ denotes the speed of light. The mapping breaks down, however, for $\epsilon$ negative (metal layers) since $\rho$ is always positive. In this work we only consider $\epsilon$ positive so that the wave is propagating in nature.

${ }^{6}$ Mathematics of stochastic methods is described by C. W. Gardiner, Handbook of Stochastic Methods for Physics, Chemistry, and the Natural Sciences (Springer-Verlag, New York, 1983). The operator $Q$ is defined as $Q f\left(\xi_{0}\right)$ $\equiv \lim _{z \rightarrow 0}(1 / z)\left\{E\left[f(\xi(z)) \mid \xi(0)=\xi_{0}\right]-f\left(\xi_{0}\right)\right\}$, with the notation $E[$ ] denoting the operation of taking expectation value. 